\title{
STEM-in-SEM: A Re-Emerging Material Measurement Approach
}

\section{Robert Keller}

NIST, Boulder, Colorado, United States

Analytical STEM-in-SEM has undergone a striking resurgence in terms of both methodology development and applications over the past 10 to 15 years, driven in part by the significant technological potential promised by low-dimensional structures such as nanoparticles and 2D materials. Key to maximizing the quantifiable information from a structure by electron microscopy is extraction of as much information as possible from every electron. To this end, characteristic mean free paths should be comparable to the size of the probed volume. A decrease in incident electron beam energy necessarily leads to shorter mean free paths for both elastic and inelastic scattering. This implies that for a given beam current within a small volume of material, use of a lower beam energy will produce more electron scattering, thereby increasing information content for potential analysis. For example, $20 \mathrm{keV}$ electrons show elastic mean free paths in the range of a few tens of nanometers, while the corresponding path lengths for $200 \mathrm{keV}$ electrons range from a few tens to a few hundreds of nanometers.

The foundational concepts for performing measurements by sending a relatively low energy ( $\lesssim 50 \mathrm{keV}$ ) electron beam through a material originated with the very early work of von Ardenne in 1938 [1], where a $23 \mathrm{keV}$ beam was transmitted through a $\mathrm{ZnO}$ crystal, revealing microstructural detail with an approximate resolution of $40 \mathrm{~nm}$ in an early form of bright-field STEM imaging. The first STEM-in-SEM system that appeared commercially became available in 1966 [2]. However, despite the early successes and demonstrations of the utility of STEM-in-SEM imaging through the 1970s [3], relatively narrow views of the analytical utility of SEM were maintained for many years [4].

During the mid-2000s, examples of STEM-in-SEM for characterization of polymers [5] and highresolution imaging of carbon nanotubes [6] demonstrated the analytical power of this measurement approach for samples considered to be difficult to characterize due to their low atomic numbers. The use of even relatively simple transmission detection schemes such as electron conversion detectors or segmented chip configurations resulted in striking, high-contrast imaging.

The current STEM-in-SEM resurgence has also been driven by recent advances in hardware capabilities that apply equally well to a SEM platform and a TEM platform. For example, the appearance of in-lens instruments [6] and transmission diffraction capabilities [7] has significantly strengthened the analytical power of the scanning electron microscope. In this context, NIST initiated several years ago a program to develop the measurement components that would be critical to the development of a fully analytical transmission scanning electron microscope, with the target of establishing metrology aimed at and optimized for quantifying individual nanostructures. The approach is to take concepts found in highenergy STEM and then adapt and integrate onto a commercial SEM platform.

Figure 1 shows a conceptual view of a future STEM-in-SEM system capable of simultaneous capture of numerous signals. A key feature of this vision is the generation of a multi-dimensional data stack comprising various forms of imaging, diffraction, and spectroscopy from each individual beam position in the raster scan, along with the development of correlative component pairs. To date, NIST has made progress in the realms of electron diffraction [7], angularly selective imaging [8], and 4D STEM [9]. Continued progress toward such a STEM-in-SEM system will require advances in elemental and/or electronic spectroscopies and multivariate hyperspectral methods. 


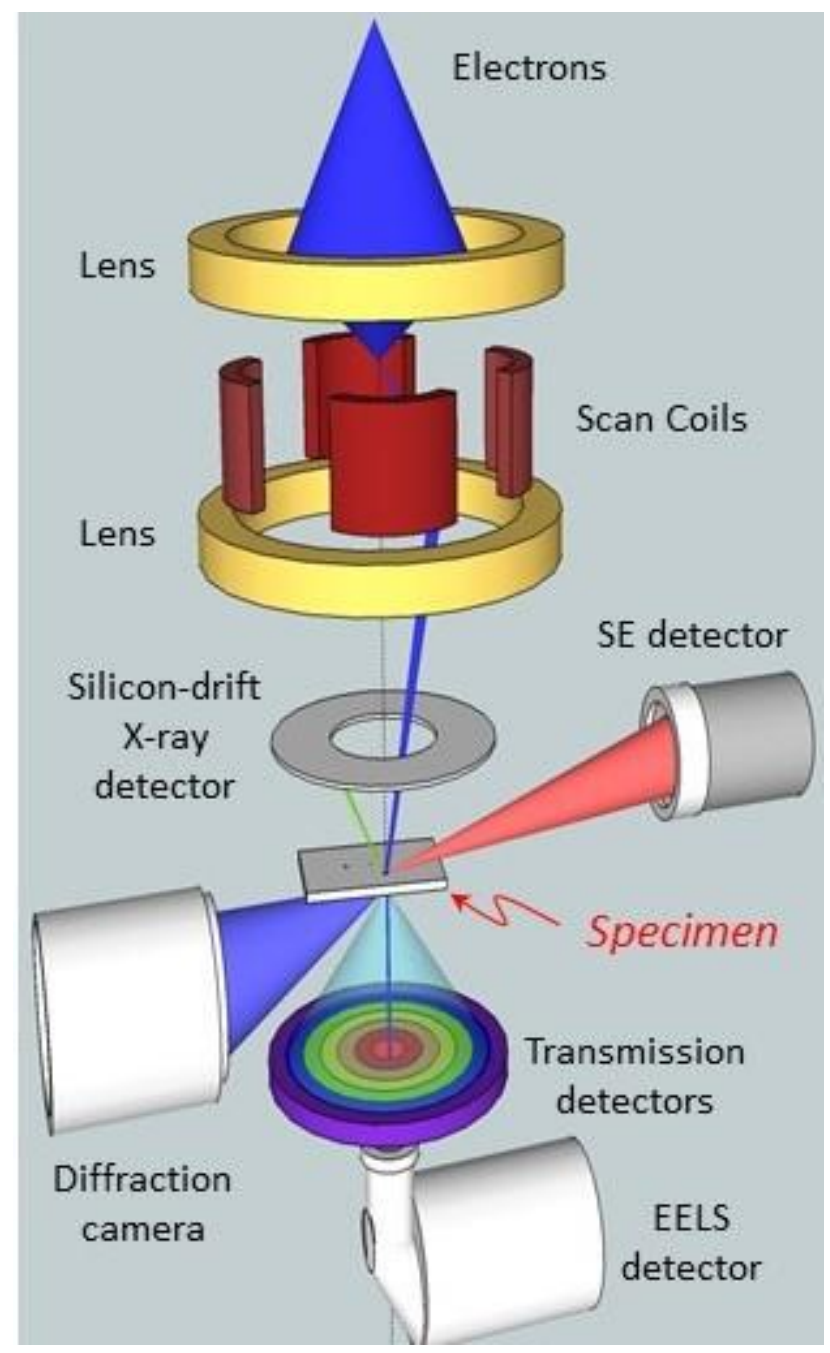

Figure 1. Conceptual view of STEM-in-SEM system with full, simultaneous imaging, diffraction, and spectroscopy capabilities.

\section{References}

[1] von Ardenne, M., Z. Phys, 1938, (108), 553-572.

[2] Klein, T., Buhr, E., Frase, C.G., Adv. Imag. Electr. Phys., 2012, (171), 297-356.

[3] Wells, O.C., Scanning Electron Microscopy, 1974, McGraw-Hill, New York, 8-10.

[4] Hawkes, P.W., Ultramicrosc., 2010, (110), 1101-1113.

[5] Guise, O., Strom, C., Preschilla, N., Microsc. Microanal., 2008, (14 Suppl. 2), 678-679.

[6] Van Ngo, V., Hernandez, M., Roth, B., Joy, D.C., Microsc. Today, 2007, (15), 12-17.

[7] Keller, R.R., Geiss, R.H., J. Microsc., 2012, (245), 245-251.

[8] Holm, J., Keller, R.R., Ultramicrosc., 2016, (167), 43-56.

[9] Caplins, B.W., Holm, J.D., White, R.M., Keller, R.R., Ultramicrosc., 2020, (219), 113137.

*This work is a contribution of the U.S. Department of Commerce and is not subject to copyright in the United States. 